\title{
REPRESENTING DIRECTIONS FOR HOUGH TRANSFORMS
}

\author{
Fabian Wenzel, Rolf-Rainer Grigat \\ Hamburg University of Technology \\ Harburger Schloßstraße 20, Hamburg, Germany
}

Keywords: Parametrization, vanishing points, direction, unit sphere, Hough transform.

Abstract: $\quad$ Many algorithms in computer vision operate with directions, i. e. with representations of 3D-points by ignoring their distance to the origin. Even though minimal parametrizations of directions may contain singularities, they can enhance convergence in optimization algorithms and are required e. g. for accumulator spaces in Hough transforms. There are numerous possibilities for parameterizing directions. However, many do not account for numerical stability when dealing with noisy data. This paper gives an overview of different parametrizations and shows their sensitivity with respect to noise. In addition to standard approaches in the field of computer vision, representations originating from the field of cartography are introduced. Experiments demonstrate their superior performance in computer vision applications in the presence of noise as they are suitable for Gaussian filtering.

\section{INTRODUCTION}

Many algorithms in computer vision operate with minimal parameterizations of directions. Some of their applications can be found in the area of stereo or multiview geometry, treating directions literally when estimating rigid body or camera motions. However, the problem of representing directions is far more general. In a projective context, it is equivalent to representing the projective 2-plane with two components. A homogeneous coordinate $\mathrm{x} \in \mathbb{P}^{2}$ is equivalent to a globally scaled version $\lambda \mathbf{x}$. Hence, when fixing the scale such that $\|\mathbf{x}\|=1$, the representation problem also becomes identical to parameterizing unit vectors or, in other terms, the surface of the unit sphere $S^{2}$.

Minimal parameterizations of homogeneous coordinates are used in optimization algorithms in order to avoid gauge, i. e. changes in the set of optimized parameters that have no effect on the value of the cost function. It has been mentioned that gauge freedoms introduce ambiguous optima and may lead to slower convergence (Morris, 2001).

Hough transforms are another example for methods that require minimal parameterizations, e. g. when locating vanishing points in an image. A vanishing point is the intersection of projected lines that are parallel in 3D space. In this context, the surface of the unit sphere $S^{2}$ acts as an accumulator space and is also called Gaussian sphere (Barnard, 1983). This way, finite or infinite vanishing points can be estimated.

Figure 1 shows an illustration of the vanishing point location problem. In this example, two projected lines appear parallel in an image. Thus, the vanishing point is located at infinity so neither can it be found in the illustrated figure nor in any larger Euclidean image. However, the two lines may be mapped onto great circles on the Gaussian sphere. It can be seen that their intersection can also be found on $S^{2}$. In particular, vanishing points at infinity can be found on its equator if $S^{2}$ is oriented such that the polar and the optical axis coincide. The described orientation of $S^{2}$ will be assumed for the rest of this work.

Numerous parameterizations of points on $S^{2}$ exist with spherical coordinates $(\theta, \varphi)$ being the most obvious one. Here, $\theta$ represents the co-latitude, i. e. the angular deviance from the polar axis, whereas $\varphi$ is called longitude. It is well-known, however, that parameterizations may either not be complete or contain singularities which makes them unsuitable for specific situations. As an example, spherical coordinates $(\theta, \varphi)$ are singular at the poles as $\varphi$ is not unique for $\theta=\left\{0,180^{\circ}\right\}$.

This paper is organized as follows: After laying out

116 


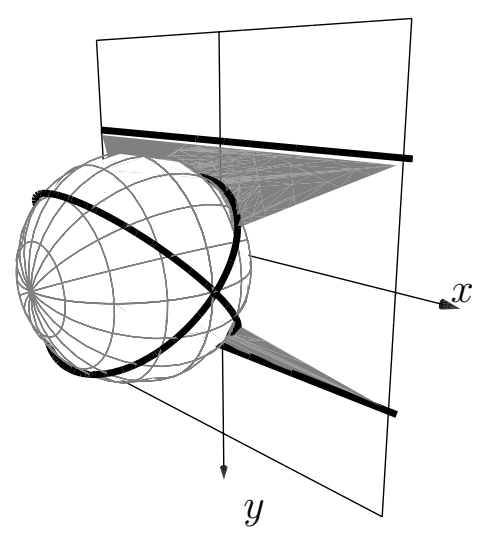

Figure 1: Gaussian sphere for detecting vanishing points: Two parallel lines intersect on the equator of $S^{2}$.

some mathematical basics, an overview and comparison of existing parameterizations of $S^{2}$ is presented. Subsequent experiments show that parameterizations originating from the field of cartography are better suited for setting up accumulator spaces for Hough maps in case of noisy input data, not only because of their numeric properties but also as they may be used for Gaussian filtering.

\section{GLOBAL AND LOCAL PARAMETERIZATIONS}

This section shows that it is impossible to have a global one-to-one parametrization of $S^{2}$. The following details are closely based on (Stuelpnagel, 1964), in which a similar explanation for parameterizing the special orthogonal group $S O(3)$ can be found.

The unit sphere $S^{2}$ topologically is a 2-dimensional compact manifold. A global 1-1 parametrization hence necessarily requires a homomorphism $h$ from $S^{2}$ to the Euclidean space $E^{2}$. A property of homomorphisms is that $h\left(U_{i}\right)$ for an open neighborhood $U_{i}$ of a point $i$ is open in $E^{2}$. Hence, $h(I)$, being the union of all $h\left(U_{i}\right)$ for $i \in S^{2}$, would still be open. On the other hand, $h(I)$ describes a continuous map of a compact space, thus is still compact. As no Euclidean space contains an open compact subset, such a homomorphism cannot be found.

Parameterizations also fail at singular points on $S^{2}$ that have infinitely many representations. However, a set of parameter patches, also called an atlas, circumvents this limitation. An atlas may contain infinitely many parameter patches. In this case, for a point of interest $i$, a unique parameter patch $h_{i}$ can be chosen. This technique is also referred to as a local parametrization (Hartley and Zissermann, 2003). A particular $h_{i}$ usually is constructed such that $h\left(U_{i}\right)$, the neighborhood of $i$, does not contain singular points and has advantageous numerical characteristics. It is interesting to note that local parameterizations still may suffer from singularity-like situations. As an example, (Hartley and Zissermann, 2003) suggests to use Householder transformations mapping a point of interest $i$ to the origin $o$ and choose a parametrization that "behaves well" in its vicinity. However, a Householder transformation does not exist if $i$ is identical to the origin and is numerically unstable if $i$ is close to it (Golub and Loan, 1996). Furthermore, some applications like the Hough transform explicitly require global parameterizations. Therefore, local parameterizations cannot be used in every situation.

A second possibility to circumvent singularities is to use an atlas with a few parameter patches only (Faugeras, 1993). Such an atlas is suitable for a projective setting as representing the complete unit sphere $S^{2}$ is not necessary. As two antipodal points $\mathbf{x}$ and its negative version $-\mathbf{x}$ on $S^{2}$ are equivalent, it is sufficient to focus on a hemisphere. Parameter patches that cover a complete hemisphere exist and are given in this paper.

\section{REPRESENTATIONS OF DIRECTIONS}

Many global parameterizations of directions are mentioned in the literature (Snyder, 1987). Some of them can be found in the field of cartography and have not been used for Hough transforms before. This section gives an overview and shows characteristics (see also table 1). Besides spherical coordinates, we concentrate on different azimuthal projections on a hemisphere. Other types are not considered in this work as they have disadvantegous properties such as singular points, computational complexity or the lack of symmetry.

- Spherical coordinates (SPHERICAL):

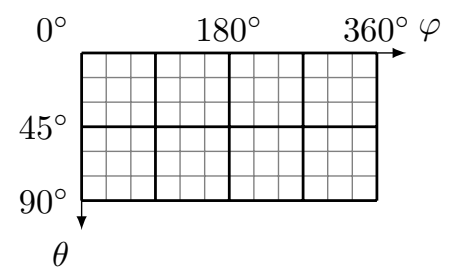

Even though spherical coordinates yield a singular point at each of the two poles of $S^{2}$, they are used 
Table 1: Overview of different global parameterizations.

Map from $S^{2}$

Map to $S^{2}$

(S): Singular points

(U): Non-unique points

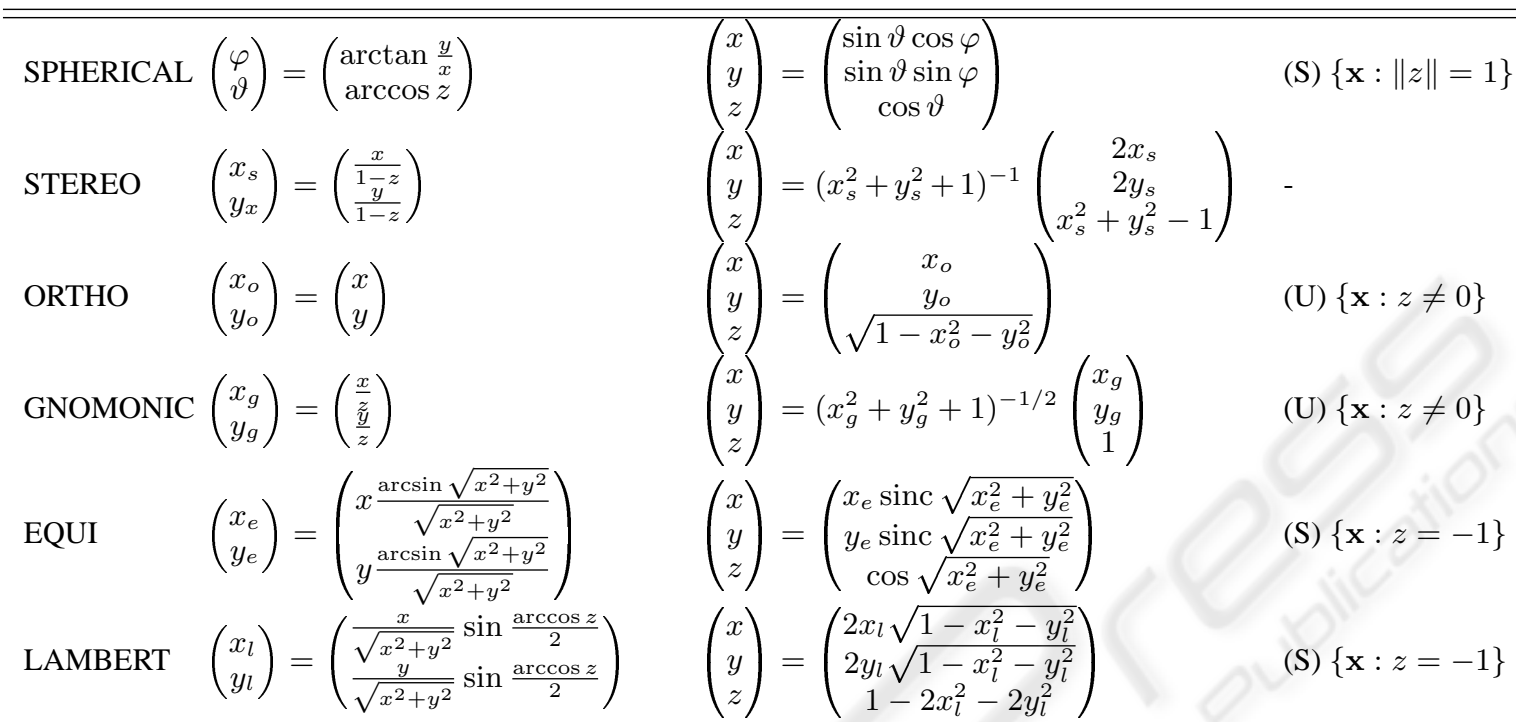

in many computer vision algorithms (Medioni and Kang, 2004). Their advantage is the simple geometric interpretation.

- Stereographic projection (STEREO):

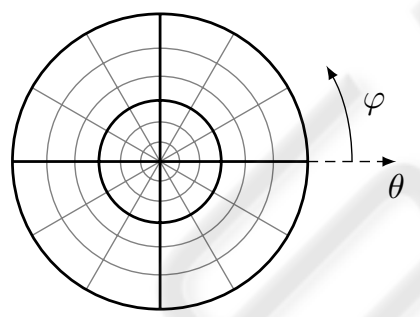

A standard approach in topology for finding a parametrization of $S^{2}$ is stereographic projection. Here, the center of projection is located at the north pole $n$, the Euclidean plane $E^{2}$ as the projection target is parallel to the equator. Corresponding points on $E^{2}$ and $S^{2}$ can be found on the same ray through $n$. It is obvious that $n$ itself cannot be mapped onto $E^{2}$. For computational purposes, a stereographic projection has the advantage of being a rational parametrization of directions in $\mathbb{R}^{3}$. Hence, it does not involve trigonometric functions.
- Orthographic projection (ORTHO):

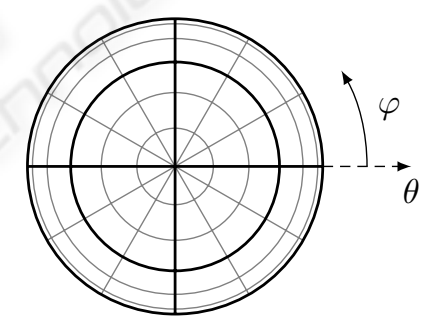

By omitting the $z$-coordinate of a vector in $\mathbb{R}^{3}$, the orthographic projection of a point can be achieved. This parametrization does not contain singularities, but an atlas is needed for representing the northern and southern hemisphere of $S^{2}$ uniquely. Its advantage is its computational simplicity given points in $\mathbb{R}^{3}$. It can be seen, however, that azimuthal resolution decreases near the equator. This drawback is important in section 4 when setting up Hough maps.

- Gnomonic projection (GNOMONIC):

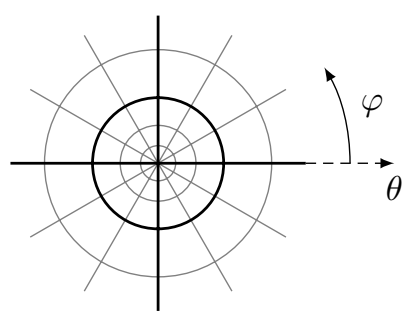


A gnomonic projection in cartography corresponds to the computation of a Euclidean representation given a homogeneous vector $\mathbf{x} \in \mathbb{P}^{2}$. Even though it is rational for points in $\mathbb{R}^{3}$, it is not unique for antipodal points and cannot find a Euclidean representation of the equator. Moreover, as the gnomonic projection inverts the process of homogenization of point coordinates, computations could directly be done on the original image. Hence, a gnomonic projection is suitable for Hough maps only if points of interest are in a camera's field of view.

- Azimuthal equidistant projection (EQUI):

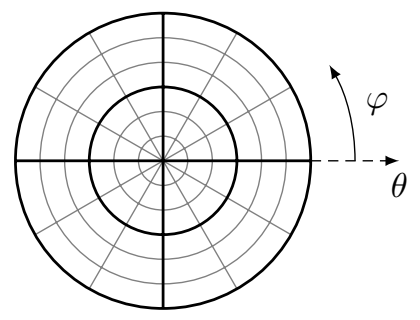

An azimuthal equidistant projection yields a 2D polar mapping of spherical coordinates. It preserves lengths of geodesics through the poles so that Euclidean distances on the map may be used as error terms.

- Azimuthal Lambertian projection (LAMBERT):

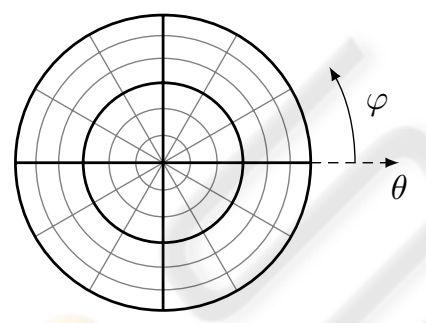

The last mapping considered in this paper is azimuthal Lambertian projection. It is area preserving, hence covariance ellipses on $S^{2}$ occupy the same area on the map. However, distances are not preserved.

\section{EXPERIMENTAL RESULTS}

Vanishing point detection via intersecting lines on a Hough map served as an application for evaluating different parameterizations. We analyzed STEREO, ORTHO, EQUI and LAMBERT. In section 3 the two remaining mappings described in this paper have already been classified not to be suitable for Hough maps: SPHERICAL yields singular points at the poles whereas GNOMONIC cannot represent a hemisphere completely. As lines in an original image are mapped onto curves on the Hough map, a polarrecursive algorithm has been used for accessing corresponding accumulator cells.

In all following configurations, the position and orientation of intersecting lines has been linearly transformed prior to Hough transformation such that the width and height of an input image does not exceed a horizontal and vertical field of view of $90^{\circ}$. As a result, the origin of the linearly transformed coordinate system coincides with the center of an image. Hence, Hough maps conform to the setup illustrated in figure 1.

A first experiment used synthetic data as input. An equirectangular point grid has been set up with line information at each position. Their individual orientations have been chosen such that all lines intersect at a single location on the $x$-axis. We examined 10 vanishing points with co-latitudes $\theta=0^{\circ}$ to $\theta=90^{\circ}$. Due to symmetry, analysis has been reduced to a single longitude $\varphi=0^{\circ}$. An example Hough map and the residual angular error between vanishing point estimates and their true positions can be seen in figure 2 . In this case errors are only caused by spatial discretization of the parameter space, i.e. by the finite resolution of a Hough map. For the used size of 255 $\times 255$ pixels, errors are below $0.5^{\circ}$ and could be decreased further by increasing the map's resolution.

In order to evaluate robustness, we added gaussian noise with a preset standard deviation $\sigma$ to all orientation angles. Figure 4 shows results for two noise levels which demonstrate the effects of the low resolution in $\theta$ for ORTHO: Starting from $\sigma=4^{\circ}$, vanishing point estimates incorrectly tend to be attracted by the equator. This problem is caused by the spatial discretization of ORTHO and can be identified in figure 3(a). Other parametrizations, e. g. LAMBERT, see figure 3(b), do not suffer from this phenomenon.

The quality of vanishing point estimates is also affected by the finite number of intersecting lines. Therefore, in another experiment, we additionally applied a Gaussian filter with kernel size $g$ to the Hough map. This approach is contrary to others in which special techniques like hierarchical (Quan and Mohr, 1989) or irregular Hough maps are used (Lutton, 1994). Results are shown in figures 5 and 6 . It can be seen that the phenomenon of attractive equator cells in ORTHO could not be resolved by Gaussian smoothing. When using other parametrizations, maximum residual errors can approximately be halved at a moderate noise level (figure 6(a)). Best results could be achieved with EQUI and LAMBERT.

A final, qualitative experiment has been done using real input data without ground truth. We used a complex-valued filter for detecting edges (Perona, 1992), (D. Fleet and Jepson, 2000) and used the phase 


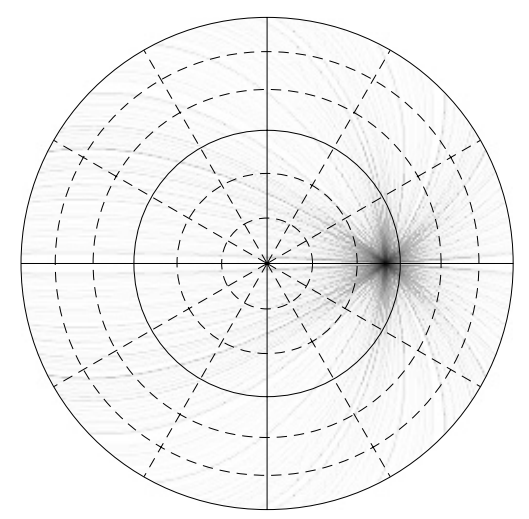

(a)

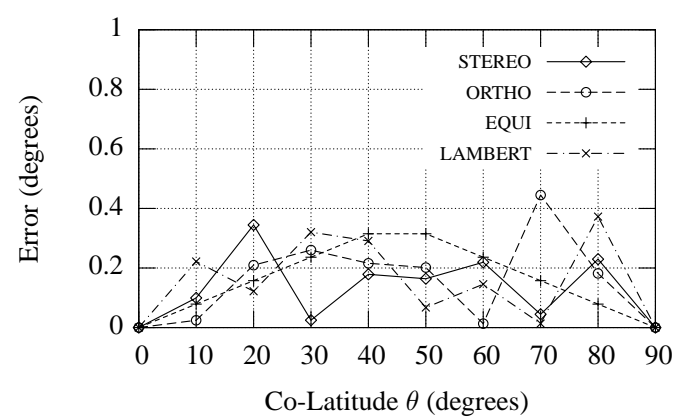

(b)

Figure 2: (a) LAMBERT map for $\theta=40^{\circ}$, (b) Residual errors due to discretization.

of the filter responses as orientation for lines on the Hough maps. Filter kernels are shown in figure 7.

Figure 8 shows a screenshot as well as a close-up of of the left vanishing point in the EQUI map. The marked locations denote point estimates before and after applying a Gaussian filter. Also in this case, estimates can be enhanced by Gaussian smoothing.

\section{SUMMARY AND CONCLUSION}

We presented parameterizations of the Gaussian sphere $S^{2}$ for detecting directions using Hough transforms. We gave an overview of both known and novel projections for the field of computer vision. Spherical coordinates as well as gnomonic projection have been identified not to be suitable for generating Hough maps. Experiments show that azimuthal equidistant and Lambertian projection yield superior results compared to stereographic and orthographic projection. Finally, we applied a Gaussian filter to Hough maps that accounts for noise.

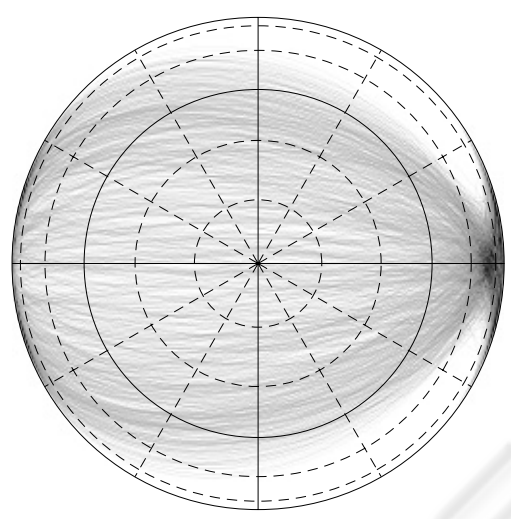

(a)

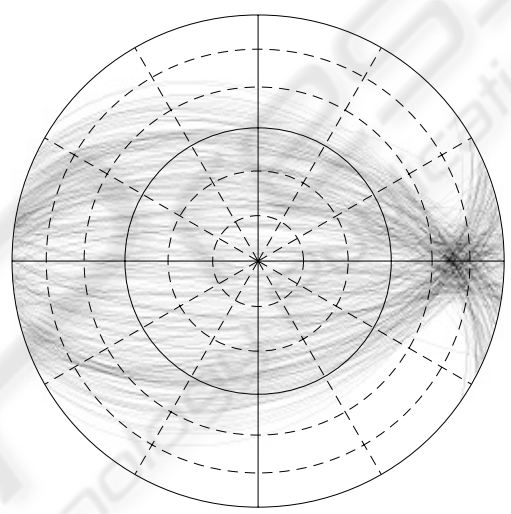

(b)

Figure 3: (a) ORTHO map for $\sigma=6^{\circ}$, (b) LAMBERT map for $\sigma=6^{\circ}$.

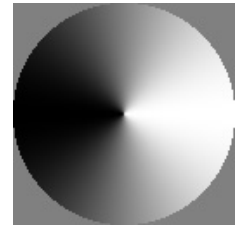

(a)

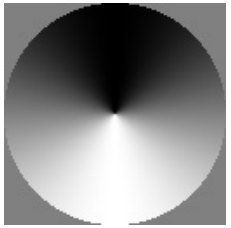

(b)
Figure 7: (a) Real and (b) imaginary part of a complex filter kernel for edges.

\section{REFERENCES}

Barnard, S. T. (1983). Interpreting perspective images. Artificial Intell., 21:435-462.

D. Fleet, M. Black, Y. and Jepson, A. (2000). Design and use of linear models for image motion analysis. International Journal of Computer Vision, 36(3):171-193.

Faugeras, O. (1993). Three-Dimensional Computer Vision - A Geometric ViewPoint. MIT Press. 
Golub, G. H. and Loan, C. F. V. (1996). MATRIX Computations. John Hopkins University Press.

Hartley, R. and Zissermann, A. (2003). Multiple View Geometry. Cambridge University Press, 2. edition.

Lutton, Maitre, L.-K. (1994). Contribution to the determination of vanishing points using hough transforms. IEEE Transactions on Pattern Analysis and Machine Intelligence, 16(4):430-438.

Medioni, G. and Kang, S. B., editors (2004). Emerging Topics in Computer Vision, chapter Robust techniques for computer vision. Prentice Hall.

Morris, D. D. (2001). Gauge Freedoms and Uncertainty Modeling for $3 D$ Computer Vision. $\mathrm{PhD}$ thesis, Robotics Institute, Carnegie Mellon University.

Perona, P. (1992). Steerable-scalable kernels for edge detection and junction analysis. In European Conference on Computer Vision, pages 3-18.

Quan, L. and Mohr, R. (1989). Determining perspective structures using hierarchical hough transform. Pattern Recognition Letters, 9:279-286.

Snyder, J. P. (1987). Map Projections; A Working Manual. U.S. Geological Survey, supersedes bulletin 1532 edition.

Stuelpnagel, J. (1964). On the parametrization of the threedimensional rotation group. SIAM Review, 6(4).

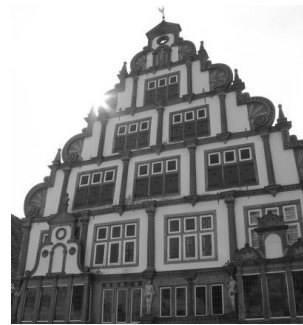

(a)

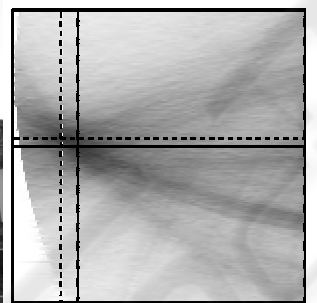

(b)

Figure 8: (a) Input image, (b) Close-up of Hough map. Dashed mark: Estimate before Gaussian smoothing. Solid mark: Estimate after Gaussian smoothing. 


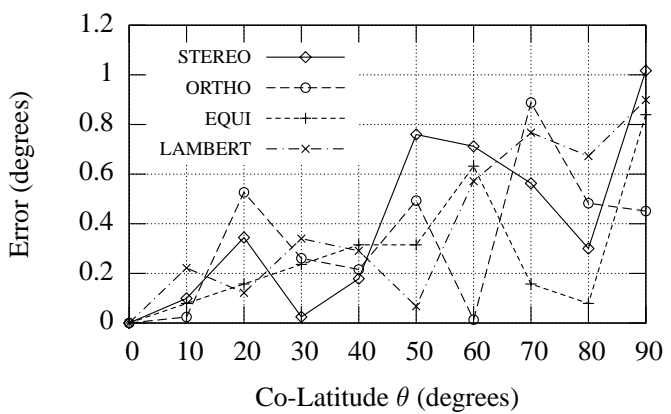

(a) Angular errors for $\sigma=2^{\circ}$

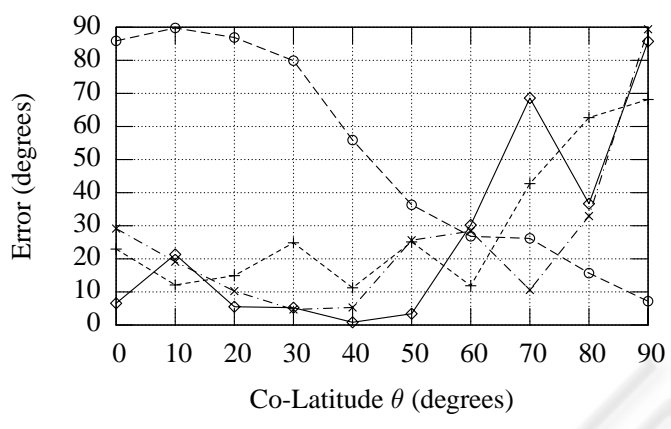

(b) Angular errors for $\sigma=40^{\circ}$

Figure 4: Effects of different noise levels.

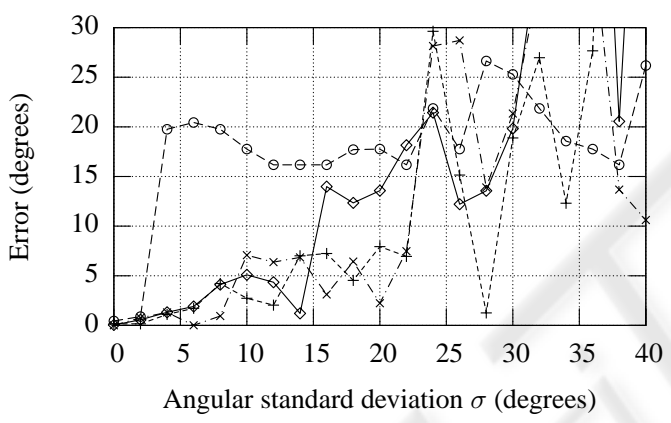

(a) Angular errors for $g=0$ pixels

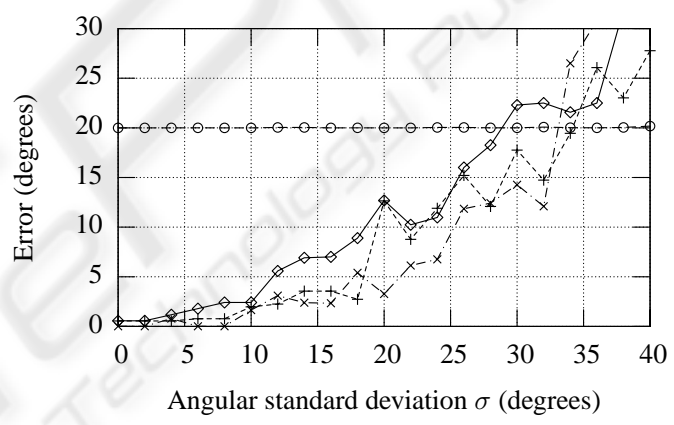

(b) Angular errors for $g=10$ pixels

Figure 5: Effects of different Gaussian filter sizes for $\theta=70^{\circ}$.

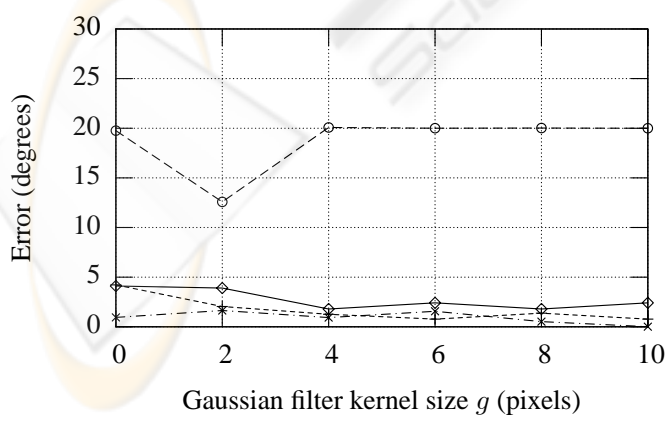

(a) Angular errors for $\sigma=8^{\circ}$

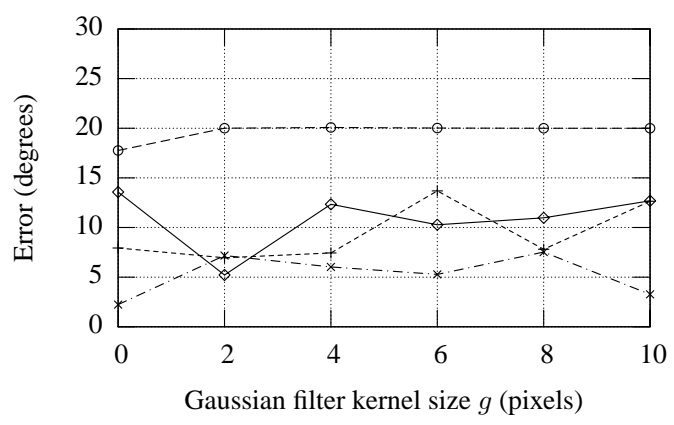

(b) Angular errors for $\sigma=20^{\circ}$

Figure 6: Effects of different noise levels for $\theta=70^{\circ}$ if Gaussian smoothing is used. 